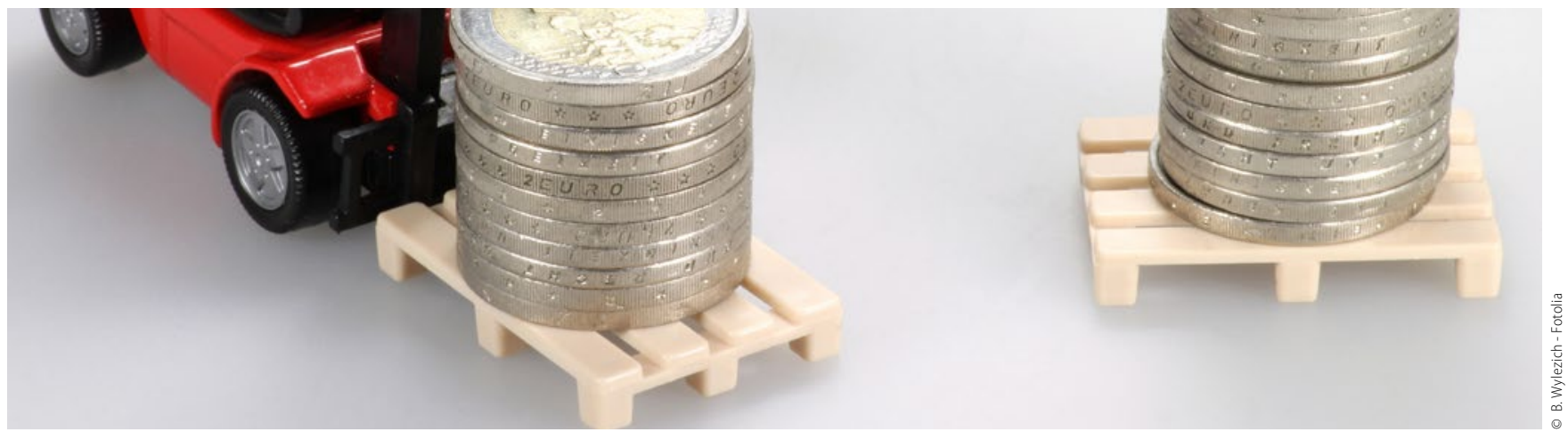

Buchhaltungs- $A B C$

\title{
K - Kostenerfassung bei der Prophylaxe
}

\begin{abstract}
Buchhaltung soll Transparenz schaffen - nicht nur für das Finanzamt, sondern auch für den zahnärztlichen Praxisinhaber. Bei sinnvollem Aufbau des praxisspezifischen Kontenplans ergeben sich aus der betriebswirtschaftlichen Auswertung hilfreiche Informationen für die Praxissteuerung. Für viele Praxen bleibt es jedoch eine offene Frage, wie rentabel das Eigenlabor und die Prophylaxe laufen. Und man versucht, über eine differenzierte Erfassung der Buchhaltung einen Überblick zu bekommen.
\end{abstract}

Die Umsätze sind ganz einfach aus der Praxisverwaltungs-Software zu entnehmen, wobei hier zu beachten ist, dass die Prophylaxe-Umsätze nicht auf den Arzt, sondern auf die Honorarerbringer geschlüsselt werden sollten, um ein klare Differenzierung zu ermöglichen.

Wie kann dagegen die Kostenseite differenziert betrachtet werden? Im Grundsatz bietet sich die Möglichkeit, sowohl für das Eigenlabor als auch die Prophylaxe separate Unterkonten im Kontenplan anzulegen. Dieser theoretische Ansatz birgt in der praktischen Umsetzung jedoch einige Tücken.

Zunächst einmal bedingt eine saubere Verbuchung die sehr intensive Vorbereitung der Buchhaltungsunterlagen. Alle Rechnungen - auch die, in denen Produkte für Prophylaxe sowie sonstige Praxisbereiche enthalten sind - müssten detailliert vorkontiert und gesplittet gebucht werden. In vielen Fällen wird aber eine eindeutige Zuordnung der Bestellungen gar nicht möglich sein. Die Materialien müssten also zwecks Analyse nach individuellen Verteilungsschlüsseln zugewiesen werden.

Ist die Verbuchung der Materialien schon aufwändig, so wäre die differenzierte Erfassung der Arbeitgeberbelastung für die Mitarbeiter der Prophylaxe und des Eigenlabors nahezu unmöglich, da sich monatlich verändernde Beträge je Mitarbeiter auch in allen Beiträgen und Abgaben finden, welche in der Verbuchung nicht aufzulösen sind.

Eine saubere Analyse ist also nur mithilfe einer zusätzlichen, auf die Buchhaltungsdaten aufbauenden Berechnung, zum Beispiel der Profitcenter-Rechnung, möglich. Bei einer ProfitcenterRechnung werden die eindeutigen Kosten und Erlöse zugeordnet, die Arbeitgeberbelastung anhand von Lohnkonten und mithilfe von Einsatzzeiten verteilt und Gemeinkosten, die nicht einzeln zugeordnet werden können, nach einem abgewogenen Verteilungsschlüssel umverteilt. Der Verteilungsschlüssel ist immer praxisindividuell zu bilden. Auf diesem Weg können für die Prophylaxe Stundenumsätze und Stundenerlöse verglichen, Stundensätze gebildet und Rentabilitäten fortlaufend beobachtet werden.

Unser Tipp: Eine genaue Analyse des Prophylaxeerfolges ist in der Finanzbuchhaltung nicht darstellbar. Die Anwendung der Profitcenter-Rechnung ermöglicht klare Zuordnungen.

Mehr Infos unter $\boldsymbol{w} w \boldsymbol{w}$.fibu-doc.de

Das in den Artikeln dargestellte Buchhaltungskonzept basiert auf der zahnarztspezifischen Buchhaltungssoftware fibu-doc und wird vom FVDZ unterstützt.

\section{Seminar}

Praxisbuchhaltung -

Leicht und verständlich

21.06.2017 Würzburg 